\title{
'It's not about money, it's about my health': determinants of participation and adherence among women in an HIV-HSV2 prevention trial in Johannesburg, South Africa
}

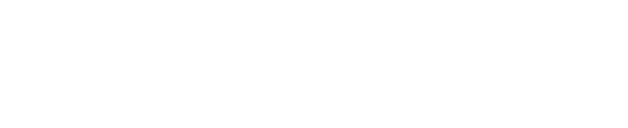

\section{Catherine MacPhail' Sinead Delany-Moretlwe' Philippe Mayaud ${ }^{2}$}

'Wits Reproductive Health and HIV Institute, School of Clinical Medicine, Faculty of Health Sciences, University of the Witwatersrand, South Africa; ${ }^{2}$ Faculty of Infectious and Tropical Diseases, London School of Hygiene and Tropical Medicine, London, UK
Correspondence: Catherine MacPhail Wits Reproductive Health and HIV Institute, School of Clinical Medicine, Faculty of Health Sciences, University of the Witwatersrand, PO Box 18512, Hillbrow, Johannesburg 2038, South Africa $\mathrm{Tel}+270$ II 3585300

Fax $+2708672446 I I$

Email cmacphail@wrhi.ac.za

\begin{abstract}
High levels of adherence in clinical trials are essential for producing accurate intervention efficacy estimates. Adherence to clinical trial products and procedures is dependent on the motivations that drive participants. Data are presented to document reasons for trial participation and adherence to daily aciclovir for HSV-2 and HIV-1 genital shedding suppression among 300 HIV-1/HSV-2 seropositive women in South Africa. In-depth interviews after exit from the trial with 31 randomly selected women stratified by age and time since HIV diagnosis confirmed high levels of adherence measured during the trial. Main reasons for trial participation were related to seeking high-quality health care, which explains high levels of adherence in both study arms. Concerns that women would abuse reimbursements, fabricate data, and share or dump pills were not corroborated. Altruism is not a primary motivator in these settings where access to quality services is an issue. This study provides further evidence that good adherence of daily medication is possible in developing countries, particularly where study activities resonate with participants or fill an unmet need.
\end{abstract}

Keywords: adherence, trial, HIV prevention, South Africa

\section{Introduction}

Much of the limited efficacy of recent biomedical HIV prevention trials has been attributed to suboptimal adherence among trial participants. ${ }^{1}$ Concerns have been raised about participation in clinical trials being driven by reimbursement and that pill dumping or sharing might be rampant in communities underserved by the formal health sector. Such motivations for participation might negatively impact on study adherence. Little research has been conducted to investigate the motivation driving participants to take part in HIV prevention, as opposed to HIV treatment research trials in low and middle income countries, and the impact this may have on trial adherence and the validity of trial findings.

In this scant literature, altruism and access to personal health and behavioral benefits have been recorded as the dominant reasons for participation in trials. ${ }^{2-4}$ Compensation was noted as important among Kenyan participants in HIV/AIDS trials ${ }^{5}$ and among vaccine trial participants in the US, Canada, and the Netherlands, although it was not one of the main reasons for participation. ${ }^{2}$ Gender differences for trial participation have been found, with women more likely than men to participate in trials for a broader range of reasons, including access to protection, services, or compensation. ${ }^{2}$ In lower and middle income countries there are particular concerns that compensation may 
drive trial participation and that this motivation may have negative consequences for adherence.

The results of clinical trials for HIV prevention are dependent on high levels of adherence, which has proven problematic for a number of prevention strategies tested thus far. ${ }^{6}$ There are three components to adherence in such trials: (1) adherence to study visits as a prerequisite for product/ intervention adherence; (2) adherence to specific products/ interventions being tested within the trial; and (3) adherence to additional practices required by the protocol (eg, the use of condoms, avoidance of vaginal douching, abstinence, etc).

Measurement of adherence is challenging. Traditionally product adherence has been measured through pill count and self-report. Biomedical markers are increasingly being used to monitor product adherence, ${ }^{7}$ but behavioral adherence continues to rely on self-reported data. Such data has inherent limitations associated with lack of participant cooperation, problems with recall, and social desirability bias. ${ }^{8}$

In this study we examined reasons for trial participation and the influence of this on subsequent adherence to study drug and procedures among female trial participants in Johannesburg, South Africa. Participants were HIV-HSV-2 co-infected women not yet eligible for anti-retroviral therapy (ART) who were enrolled in a randomized, double-blinded, placebo-controlled trial to examine the efficacy of twice daily HSV-2 suppressive therapy with aciclovir in reducing HIV transmissibility. ${ }^{9}$ Through this study we hope to confirm high levels of trial adherence and how reasons for participation might influence this. This information may be useful in the design of future trials of HIV prevention interventions.

\section{Methods}

\section{Study participants}

Between April 2005 and April 2006, 300 HSV-2 and HIV seropositive women were enrolled in a trial and randomized to receive aciclovir $400 \mathrm{mg}$ one tablet twice daily $(\mathrm{n}=152)$ or matching placebo $(\mathrm{n}=148)$, and followed up at monthly intervals for 3 months. Women had a mean age of 32 years and a 1 year median time since HIV diagnosis, and were compensated ZAR150 (approximately US\$20) for each study visit. Trial outcomes have been reported elsewhere. ${ }^{9}$

In the current study, a $10 \%$ random subsample of trial participants from both study arms $(n=31)$ was recruited according to three criteria: age (women were selected from the 18-30 years and 31-60 years age groups); duration of HIV infection (whether women were aware of their status since $\leq 1$ year or $>1$ year); and willingness to participate. Once trial exit visits were completed, contact information collected during the trial was used to establish interest in participating in an additional study visit and to set up interview times with participants. Appointments were made for women to visit the clinic at a time suitable for them. At this visit, the objectives of the in-depth interview (IDI) visit were further explained and women provided written consent for their participation in the interview, as well as permission for their discussion to be tape recorded. Women were compensated ZAR50 (approximately US\$7) for their participation.

\section{Trial procedures and quantitative data collection}

During the trial, participants were seen at monthly clinic visits (visit interval 30-35 days) for a total of three visits, when drug packs were returned for pill count, a new supply of drugs dispensed, and data on self-reported adherence collected. At each visit, participants received adherence counseling which included instructions for study drug use, storage, and handling of missed doses.

All participants received pre- and post-test sexually transmitted infection (STI)/HIV counseling, regular risk reduction counseling, free condoms, treatment of STIs, and referral to local HIV treatment centers. All participants with symptoms of genital ulcers received presumptive treatment for genital herpes with aciclovir for 5 days, irrespective of study arm, in addition to the standard antimicrobial regimen of syndromic management. As required by the study protocol, participants were counseled to avoid vaginal cleansing and unprotected sexual intercourse for 72 hours before their next clinic visit when genital samples were collected. Where sex was unavoidable, participants were encouraged to use a condom in order to avoid contamination of samples with semen. Free condoms were provided.

\section{Qualitative data collection}

Interviews were conducted face-to-face with a single female interviewer in local South African languages. The interviews lasted approximately 90 minutes and were tape-recorded. The interviewer used a semi-structured interview topic guide in which women's reasons for participation and their adherence to various aspects of the study were examined. The interview topic guide was developed in English and reviewed by the interviewer and study investigators to ensure that meaning in local African languages was clear and that there was agreement on correct terminology for discussions. To ensure that participants were comfortable discussing the issue of trial adherence, data collection was conducted after each participant's exit visit from the trial, once adherence 
measures had already been calculated and participants were no longer on treatment. Additionally, women were assured of confidentiality and explicitly informed that their adherence issues would not be discussed with staff from the trial.

\section{Qualitative data analysis}

Recorded interviews were independently translated and transcribed into English. One of the investigators (CM) read through all transcripts to identify major themes. Thereafter a coding frame was constructed using Atlas.ti (v 5.2; Scientific Software Development, Berlin, Germany) and a line-by-line review of transcripts was conducted. Quotes were allocated to codes using a framework analysis approach. ${ }^{10}$ Text corresponding to each of the themes was grouped together and reviewed. Analysis continued as an iterative process through discussions and refining of the major themes. Sub-themes were then established using a method of constant comparison $^{11}$ to break down higher-level codes (themes) into smaller categories within the framework. Quotes were selected for inclusion in the paper if they reflected dominant attitudes and actions or were good illustrations of counter normative behavior.

\section{Ethical approval}

The Human Research Ethics Committee (HREC) of the University of the Witwatersrand and the ethics review board of the London School of Hygiene and Tropical Medicine approved the trial. The trial was registered with the South African Department of Health national register of clinical trials (DoH-27-0207-1376). The qualitative interview was approved by the HREC as an auxiliary study to the trial (Reference: 061003).

\section{Results}

\section{Reasons for participation in a clinical trial of HIV prevention}

In order to begin understanding adherence in this trial, women were asked about their reasons for participation. The vast majority of participants first approached the clinic and became involved in the trial in an attempt to get information and to gain control of their health, regardless of the time since their HIV diagnosis.

[I came to the clinic] to get information - I came to the clinic to sort out my health. (Participant \#15)

Working with women's interest in their health, trial screening staff at the clinic encouraged women to investigate whether they were infected with HSV-2 and HIV. A number of women in the trial noted that the opportunity to establish whether they were HIV and HSV-2 seropositive were important motivators in their trial participation. These women had not necessarily experienced symptoms of either disease prior to screening and enrolment into the trial but were aware of the high prevalence of both in local communities, either prior to engagement with trial staff or after participation in screening health talks, and wanted to be sure of their own serostatus.

The reasons that I came here is that they told us that they are going to check us first if we do not have herpes. Then I thought it is better that I come because herpes is something that is not nice to have. The other thing is the sooner they find out that I have herpes, the sooner they will treat it. It is worse when you are HIV positive because there are many things that can happen without [you] knowing, like infections. (Participant \#14)

The thing that made me decide to come here is because of family planning. Then I found the Community Health Worker talking down there in the family planning area and saying how it is important to do a [HIV] test and to come to check for herpes. Then I told myself to come and find out about my health because it is not good if I do not know what is going on with my health. (Participant \#11)

Others noted that information on the trial was provided to them at the time that they had approached public sector clinics for STI treatment. These women were concerned specifically about their sexual health and used participation in the trial as a means to better understand the symptoms that they had.

The reasons to participate in the trial are because I heard about herpes and I used to have a rash in my vagina. Then I thought the treatment maybe would help me. (Participant \#12)

The thing that made me decide to participate in the study was I started to see symptoms that I did not understand, like I had discharge and I was always sick so then I decided to go and find out about my status. (Participant \#16)

A further group of women joined the trial already aware of the fact that they were either HIV or HSV-2 seropositive (or both). They noted that participation in the trial gave them the opportunity to better manage their illnesses and to obtain additional information about infection, treatment, and the prevention of transmission to others.

The first reason was that I wanted to check myself, what is my health like and I wanted them to treat me because I have herpes. Those were the reasons why I came to [clinic name]. (Participant \#8) 
The people from [clinic name] came to [local community] and told us about the clinic so then I told myself that I must come to the clinic to find out more about my health. Even though I knew that I am HIV-positive but I wanted to know more about herpes. (Participant \#25)

At the time of the trial, aciclovir was not available as first-line therapy for genital ulcers in the South African public health sector, and could only be obtained at considerable cost through the private sector. Among those women who noted that the potential for herpes treatment was a strong motivator for participation, a small subset particularly noted that free treatment had motivated their participation. Participant \#4 noted: ' $\ldots$ they said after finding out that you have this virus they will give you free treatment.'

A strong motivator for trial participation among this group of HIV-positive women was the ability, through the trial procedures, to have monthly updates on their CD4 cell count. At the time of the trial, antiretroviral treatment of HIV was available free in the public health sector for patients with CD4 cell count below 200. Women who had known their HIV-positive status for more than 1 year were therefore particularly interested in participating in the trial to obtain more regular CD4 testing than would usually be available to them in the public sector, and thereby increase their chance of initiating HIV treatment as soon as they were eligible.

I have not been sick since I knew that I am HIV-positive but I know that if you are HIV-positive you must always check your CD4 count, you must not wait until your CD4 count goes down. I think that is the thing that made me decide to come and join the study even though they told me that they are doing research but I wanted to see if they were going to help me. (Participant \#19)

Although women did discuss HIV and HSV-2 specifically during the interviews, they were more likely to speak of wanting to improve their health more generally. This reflects that women's decisions to participate in this clinical trial were made independently of their diagnosis and reflect an attempt to address concerns with their health generally, rather than being specifically focused on HSV-2 and aciclovir. Although access to information and treatment dominated discussions, a small number of women discussed other motivations for trial participation, beyond access to improved health care.

The issue of receiving reimbursement was not a motivating factor for most women. In fact, only a single participant noted that reimbursement had affected her decision to participate in the trial when she said that beyond the benefit of having her CD4 cell count monitored: 'I would say money also helped me in addition to the other things that I was getting here.' (Participant \#14). Indeed, if women did discuss the issue of reimbursement, it was more likely to be in the context of assuring the interviewer that money had not been part of their motivation for joining the study. Participant \#20 stated: 'I joined the study because I wanted to know about my health, it was not because of money.' This type of statement was echoed by a further participant.

My aim for coming to the clinic was to get something to help me. I found what I was looking for because the medication that I received helped with my problem. My aim was not to receive money when I came here. If it was just because I wanted money then it would have been better if I had just stayed at home. (Participant \#16)

Altruism was also not a major determining factor in women's trial participation. Most women displayed a good understanding of the concepts of research trials, randomization, and placebo controls. The statement by Participant $\# 19$ : 'I was wishing to get the one that they said it is the active one. Then I told myself that what about the nurse? She doesn't even know which one is going to work. So I thought whatever I am going to get will be fine with me' reflects the attitude of most women in the trial. Women reported feeling certain that whatever drug they received in the trial would assist them, although they understood that randomization and placebo drugs were a necessary component of the research process.

While this detailed understanding of the mechanisms of randomized placebo-controlled trials might suggest that women's participation was motivated by altruism, only a single participant explicitly noted that her decision to participate was motivated by a desire to assist the research process. She explained:

The thing they told us is that they don't know if it is working or not because they are still doing research, so I did not have a problem if it is not working because I wanted to help them to discover the information. (Participant \#4)

Overall, women discussed their decisions to participate in the research process as a rational health decision in the face of general poor reproductive health in their local communities. This is reflected by the fact that many of the women reported having discussed the trial with family and friends when making their decision about participation.

My mother helped me to make the decision to come to the clinic. She told me that I must go-maybe it's where I am going to get help, because I was starting to stress myself. (Participant \#6) 
Women were most likely to have asked for opinions on participation from female relatives or friends, and while most reported that their partners were aware of their participation, few mentioned that their male partners had assisted them in deciding to participate in the study. Additionally, women specifically reported discussing trial participation with friends or family they knew were infected with HIV or HSV-2 in the hope that they too might make the decision to participate in the study.

I did tell people about the study; like that they do HIV and HSV-2 tests. The reason for me to tell them is that they have sores and that problem with their vaginas. I also told them that I used to have a sore in my vagina but since I have enrolled in the study all the sores are gone. (Participant \#3)

There were some concerns about women being actively dissuaded from trial participation given the stigma surrounding HIV in South African communities and a degree of mistrust of medical research based on historical exploitation by the apartheid government and perceptions of profit motives of large pharmaceutical companies. However, most women interviewed here said that there were no individuals who had attempted to stop them from participating in the trial, although some noted that their partners had not supported their decision to enroll.

Interviewer: Did you have a problem with other people seeing you coming to the clinic?

Respondent: No, I did not think about that because you know as a person you must not think about what other people are going to say if they see you going to that clinic. (Participant \#8)

Given that women perceived participation in the trial as an important and beneficial health intervention, most reported that they ignored negative attitudes from partners. Additionally, women did not appear to be concerned with being identified as trial participants. Indeed, many specifically said that the trial was about their health and that they would not have paid attention to anyone who had attempted to sabotage their participation.

\section{Adherence to trial visits}

HIV prevention trials in general and this trial specifically, have three adherence components. First, adherence to study visits; second, adherence to the study drug (in this case aciclovir or placebo); and third, adherence to particular behavioral requirements.
Visit adherence is an important component of study drug adherence because participants needed to visit the study clinic every month in order to receive new drug supplies and provide data on product adherence. During the trial, adherence to clinic visits was considered good. Overall, more than $90 \%$ of planned study visits were completed 541/604 $(90 \%)$ in the aciclovir arm and 545/592 (92.1\%) in the placebo arm. During post-trial qualitative interviews, one-third of participants mentioned having missed scheduled visits, although most noted that they had made up the visit within the following week.

No reasons for missed visits emerged above all others; problems with work, childcare, travel plans, and simply forgetting were all mentioned.

Yes, I was supposed to come on Wednesday and then I did not come on that day, I came on the next day because my mother was not at home so there was no one to look after my baby. (Participant \#15)

Two women mentioned that they had missed their scheduled visits due to menstruation. This was, however, a protocol requirement and was accommodated in the visit schedule. In general, women noted that there were few good reasons for missing clinic visits: visit schedules were known ahead of time and required sufficient planning. Such planning included having small children accompany them on study visits when necessary, ensuring that they had transport money on the day, and marking appointments on the calendar so that they were not forgotten.

The strongest factor encouraging clinic visit adherence was staff support. This was mentioned by more than half of the interviewees and was often repeated multiple times in individual interviews. Women consistently noted that the clinic staff had been friendly, concerned, and had provided guidance and assistance with problems beyond adherence to the study.

I think it [staff attitude] helped me because when I thought about coming to the clinic I knew that I am going to be laughing and talking to people who care about me and I loved to come to the clinic. (Participant \#19)

Women commented on this particularly, given that they were HIV-positive and seemed to expect some degree of poor treatment from clinic staff, as illustrated by Participant \#8 who noted that 'They [clinic staff] helped me because they managed to sit down with me and they said that this is not the end of the world, you can continue with your life and when I told them about my status they did not discriminate 
against me or talk negatively about me.' In addition, study staff assisted with adherence through reminder phone calls and flexibility around study visits when participants had scheduling conflicts.

As well as reminding participants about their medication, family were also an important source of encouragement for visit adherence, although this was discussed less frequently than as motivation for product adherence.

My sister used to encourage me and remind me about my

date to come to the clinic ... Everyone in my house used to

encourage me to come to my study visits. (Participant \#6)

Discussion of barriers to visit adherence was relatively limited, possibly as a result of the fact that women were interviewed when they had completed all study visits. The most significant barriers to participation were issues with transport (8/31 interviewees), with women citing poor transport infrastructure and financial hardship as impediments to visit adherence, although most managed to borrow money for transportation costs knowing that they would be reimbursed after each clinic visit.

The thing is I had to borrow money for transport so that I can come to the clinic ... when I get home I must pay the money that I borrowed before I came to the clinic by using the money from here. (Participant \#17)

There was a transport problem because if I come here I must change taxis twice so that is a problem ... It is far, but there is nothing that I can do about that. (Participant \#23)

A further challenge that women perceived to their visit adherence was waiting times at the clinic (5/31 interviewees), despite this being vastly improved over normal waiting times in public health clinics. A small number of women also mentioned that their employment was a barrier to their participation in the trial (this in connection with long waiting times in the clinic). Most employed women were however accommodated, where possible, through early morning appointments and Saturday clinics.

\section{Adherence to trial product}

Pill counts were seen as a relatively objective means of measuring adherence to study product between visits, and adherence was calculated as the proportion of pills taken out of the total number of pills that should have been taken during the visit interval. Pill count measures provided a useful estimate of average adherence each month, but relied on participants to attend scheduled visits and to remember to returned used pill packs and remaining pills. Pill pack return rates (ie, the proportion of pill packs returned to the clinic out of those dispensed at the previous visit) were similar when comparing the aciclovir group with the placebo group at month 1 (96\% vs 92\%), month 2 (93\% vs 96\%), and month 3 ( $94 \%$ vs $92 \%)$.

In this trial, levels of $>90 \%$ adherence to the product were desired. These levels of adherence were observed in the early trials of aciclovir where suppression of $>80 \%$ of HSV-2 reactivation was achieved. ${ }^{12,13}$ It was thought that similar levels of adherence would be required to see an impact on HIV transmissibility. Using pill count as a measure, overall adherence was high, with a mean adherence rate of $95 \%$ in both study arms among those who returned their pill boxes.

In addition to pill counts, adherence was also measured by self-report. These complementary measures provided additional information on the number of consecutive doses (ie, one tablet twice a day) missed. HSV-2 has been shown to resume reactivation after 5 days of stopping HSV-2 suppressive therapy and so it was important to know about the number of consecutive doses missed in addition to an overall estimate of adherence by pill count. ${ }^{14}$ At each visit, participants were asked about missed doses and reasons for this. Participants who reported missing 6 or more consecutive days at each of the monthly visits were considered to have lost any benefits of HSV-2 suppressive therapy. Overall, slightly more participants in the placebo arm compared to the aciclovir arm reported missing 6 or more consecutive doses at all visits except the exit visit (Table 1). The median number of doses reported as missed at each month was also higher among the placebo group. Forgetting to take pills, missing visits, and being away from home were the three most frequently reported reasons by women for missing doses in both treatment arms. Side effects were seldom reported as a reason for omitting to take drugs. Pill sharing was not reported as a reason for missed pills by participants (Table 1), although in a specific question about pill sharing, one participant who was in the placebo arm reported at her month 3 visit that she had shared ten pills with another person.

During the post-trial interview discussions about adherence, almost half of participants mentioned that they had missed some doses of their study drug. In most instances, specific reasons for missed doses were not provided and the number of missed doses was not detailed. In all cases, participants noted that they had missed 'a couple of doses' and had used various strategies to make up missed medication. 
Table I Self-reported adherence measures and reasons for missed doses in the trial

\begin{tabular}{|c|c|c|}
\hline & $\begin{array}{l}\text { Aciclovir group } \\
N=|5|\end{array}$ & $\begin{array}{l}\text { Placebo group } \\
N=148\end{array}$ \\
\hline \multicolumn{3}{|c|}{ Missed six or more consecutive doses } \\
\hline Month I & $17(11 \%)$ & $15(10 \%)$ \\
\hline Month 2 & II (7\%) & $15(10 \%)$ \\
\hline Month 3 & II (7\%) & $15(10 \%)$ \\
\hline \multicolumn{3}{|c|}{ Median number of doses missed (IQR) } \\
\hline Month I & $6(3-12)$ & $4(2-7)$ \\
\hline Month 2 & $5(2-8)$ & $8(3-16)$ \\
\hline Month 3 & $3(2-6)$ & $16(6-24)$ \\
\hline \multicolumn{3}{|c|}{ Proportion of women reporting following reason for missing doses ${ }^{a}$} \\
\hline Missed visit & $21(14 \%)$ & $23(16 \%)$ \\
\hline Forgot & $44(30 \%)$ & $32(23 \%)$ \\
\hline Away from home & $15(10 \%)$ & $12(8 \%)$ \\
\hline Change in routine & $2(1 \%)$ & $6(4 \%)$ \\
\hline Lost pills & 0 & $4(3 \%)$ \\
\hline Experienced side effects & $2(1 \%)$ & I (I\%) \\
\hline Illness & $3(2 \%)$ & $6(4 \%)$ \\
\hline $\begin{array}{l}\text { Shared pills with } \\
\text { another person }\end{array}$ & 0 & 0 \\
\hline Other reason & I (I\%) & $4(3 \%)$ \\
\hline
\end{tabular}

Note: ${ }^{\text {TTh }}$ This a cumulative measure which represents the proportion of women who cited a particular category as the reason for missed doses in a particular month, summed over the 3-month period.

Abbreviation: IQR, interquartile range.

It was alright and then there were some days where I forget to take my medication but not for such a long time. It happened that I would not take my medication in the morning but I will take two in the evening. (Participant \#13)

The reasons for drug non-adherence provided in the interviews closely matched self-reported information collected quantitatively during the trial. In most instances participants noted that a change in routine and traveling were reasons for missed doses, as illustrated by Participant \#20 who mentioned that '... traveling was the thing that mostly disturbed me in taking my pills.' Other participants noted similar issues with being away from home.

I used to have a problem with taking my pills because I used to visit my boyfriend then I would often forget to take my pills with me. (Participant \#27)

A small number of participants mentioned that they had experienced some negative health impacts that they attributed to the study drugs. One participant noted that the headaches she experienced during the trial prevented her from taking all doses but other side effects attributed to the study were minor and were not reported as impacting on adherence.
Half of the interviewees specifically noted that they had not missed any doses of study drug. There were no instances in which the participant noted both and this therefore seems an accurate representation of product adherence.

Yes, I did take my pills the way the way they told me to do. Like they said I must take one in the morning and one in the evening. So [name], the person who was working at the pharmacy told me that it's better if you take both at the same time so I would not forget to take them ... I never missed to take my treatment and I never missed my clinic visit. (Participant \#15)

A small number of interviewees explicitly linked their motivation to adhere to study drugs to a perception that their health had improved since enrolment in the study and to ensuring that they experienced the health benefits they believed would accrue through taking the correct dosage. Participant \#9 mentioned the following: 'I think it's because I started to feel better and there were some changes so I continued taking the pills.' This concern with health benefits was reflected in participant comments about honesty in reporting adherence to clinic staff. Participants reflected that lying about their adherence would limit the degree to which they could measure health improvements or manage health problems.

I was honest because I wanted to know what was happening to me if I forgot to take my pills or whether I have improved or gotten worse [gained or lost something]. (Participant \#16)

I think to lie is not good - it's better you tell them the truth even if you forgot to take your pills. Moreover when you lie you are lying to yourself because if you did not take the pills and then if hide this without telling the nurse it means you are killing yourself. (Participant \#19)

The study specifically examined whether pill sharing might account for poor adherence. Among the participants interviewed after the trial, no participants reported pill sharing and many noted that it was an unwise strategy if one was concerned about one's own health and that of partners, family, or friends. Participant \#14 noted: 'I did not share with anyone because if they give you some pills it is for you, not for other people.'

Participants reported a range of strategies that assisted them in maintaining adherence. Specifically, participants reported taking missed doses in the evenings (as detailed above) and particularly receiving support from family, friends, partners, and clinic staff. 
I was honest because I told them [clinic staff] I was taking my pills and I was honest about it. The other thing is that I never missed to take my pills and my husband used to set the alarm so the time it started to go off I knew it was the time for me to take my pills. (Participant \#19)

There were few instances in which participants mentioned other strategies for adherence other than assistance from family, partners, or clinic staff. One participant noted that she made sure to always carry the medication with her in her bag and another mentioned that she used her cell phone reminder function as an alarm for her scheduled medication.

\section{Adherence to trial behavioral requirements}

The trial protocol required that participants self-collect vaginal tampons and refrain from vaginal practices such as douching. Participants were also counseled about condom use for general safe sex, but particularly for sex acts immediately prior to study visits that might contaminate genital shedding samples. Most women in the study were experienced tampon users and most did not report douching prior to enrolment in the trial. In instances where women did practice douching or other vaginal cleansing processes, the explanations provided by study staff were viewed as sufficiently clear to change their practices.

I did not have a problem about douching because they told me that it is not good to insert your finger in your vagina.

(Participant \#26)

Adhering to consistent condom use was the greatest adherence challenge for women, although whether this was in the context of general transmission prevention or to avoid genital sample contamination was not detailed by participants. Among those who struggled with adherence to condom use, women indicated that partners suggested that condom use implied a lack of trust, as illustrated by Participant \#11 who stated: 'Yes I did have a problem with my partner - he did not want to use condoms. When I asked him to use condoms, he said it means I do not trust him, you see.'

In some instances women chose to frame discussions of condom use within the requirements of the study and being necessary for their health. These women appeared to have some success with increasing condom use within their partnerships.

Yes, it helped me because sometimes he [partner] would want to have sex with me without using a condom. After coming here it helped me because since I told him we must use a condom when we have sex he listens to me and I also take my pills nicely... The thing that helped me too much is to use a condom because I did not have any infections. When my husband wanted to have sex with me I said we must use a condom and before he used to say today we must not use a condom. Then I would agree with him, because I told myself that we are both HIV positive so it's better that we just finish ourselves. But after getting these pills and when the sister explained to me, then I learned more that you can prevent some of the things that will harm you.

(Participant \#10)

Others noted that the information they received during study visits empowered them to make decisions about their health and were therefore able to end relationships in which partners remained unwilling to use condoms, such as Participant \#12 who indicated: 'It was a problem, that is why I stopped seeing him because he did not want to use a condom, and he did not believe me, he was saying I am telling him lies. I realized that if he does not want to use a condom he is going to re-infect me so it's better that we stop seeing each other.'

\section{Discussion and conclusion}

This study confirms observations in the randomized, doubleblind, placebo-controlled trial that adherence to the study visit schedule, study product, and protocol requirements was generally very high. The measures used for recording product adherence during the trial were complementary in the information that they provided and were corroborated during in-depth interviews with participants after the trial. Ultimately, the outcomes observed in the trial confirm that the adherence levels reported during the trial are likely to be reliable. ${ }^{15}$

Evidence from the interviews reinforced the notion of participant agency through using the trial to take control of their health and to access optimal health care in a setting of uncertain health care quality. This participation motivation explains the high levels of product adherence seen in both arms of the study. Much has been written about the poor quality of health care in the South African public sector, specifically with regard to the attitude of health care providers. ${ }^{16,17}$ Participants in this trial made use of the availability of well-trained and accessible staff to gain information about their disease status and to effectively manage their HIV prior to initiating antiretroviral therapy. Qualitative research with women participating in other HIV prevention trials has highlighted the importance of access to good quality health 
care and caring staff as motivators for trial participation, ${ }^{18,19}$ although this study is the first to tie these aspects to good adherence.

Participation in clinical trials creates uncertainty through the use of drugs with unknown efficacy or an inability to determine whether one is receiving the active or placebo drug. ${ }^{20}$ In this instance, women understood that they might be receiving an inactive product, yet were willing to engage with the trial nonetheless. Aciclovir for the suppression of herpes was not available in the South African public health sector at the time of this study and while participation may have reflected a need to access long-term HSV-2 treatment, participation in the trial went beyond access to a single drug. Rather, participation was premised on access to good health care in the context of HIV more generally.

Concerns that women would abuse reimbursements, fabricate data, share pills, or dump pills do not seem to have been confirmed. Women appeared to have been honest about their adherence limitations, particularly with regard to condom use. Results may have been different had the trial targeted primary HIV prevention, although barriers to condom use are significant even with intensive safer sex counseling resources allocated to clinical trials. ${ }^{21,22}$ While the negative influence of partners on condom use should not be underestimated, in this context, condom use adherence may not have resonated with women's desires for health promotion as much as the potential to receive aciclovir for the management of their herpes infection.

While the limited literature suggests that altruism and compensation play a role in study participation, ${ }^{2,4,23}$ these were not primary motivators in this case. Although the key outcome of the trial was the prevention of HIV transmission through limiting HIV shedding, no participants framed their participation in an altruistic desire to prevent further HIV transmission.

This study provides further evidence that good adherence to daily medication is possible in low and middle income countries characterized by poverty and social deprivation. High levels of adherence have previously been demonstrated for anti-retroviral use in South Africa, ${ }^{24}$ but this study indicates that high levels of drug adherence for non-life threatening diseases within the uncertainties of a clinical trial can also be achieved when study activities are highly applicable to participants or fill an unmet need. While there are additional complexities associated with primary HIV prevention trials in healthy volunteers, the results of this study bode well for trials of pre-exposure prophylaxis (PrEP) in sub-Saharan Africa. Additionally, the study highlights adherence as a multi-component issue extending beyond study product to visits and behaviors associated with trial procedures. The study indicates the additional value of conducting multiple data collection procedures using different techniques for data triangulation.

\section{Limitations}

Given that the qualitative component of the study was conducted at the completion of the main clinical trial, the study may have excluded women who were lost to follow-up in the main study and who may have experienced greater barriers to participation. This influence is likely minimal given than only 28 women missed their final month 3 visit in the trial. Although not supported by the quantitative data collected during the trial, there may have been some social desirability bias to report higher levels of study adherence than actually experienced. Attempts were made to minimize this bias through conducting qualitative data collection at the end of the trial and using different staff to those involved in the collection of trial adherence data.

\section{Acknowledgments}

The authors wish to thank the volunteers for their participation in the study and acknowledge Zethu Mchunu for assistance in conducting interviews with trial participants. The main study was supported by grants from the Wellcome Trust (GR074151MA), the South African National Research Foundation (TTK2005071300016), and the UK's DFIDfunded Knowledge Programme on HIV/AIDS and STI of the London School of Hygiene and Tropical Medicine. This ancillary study was investigator initiated.

\section{Disclosure}

The authors report no conflicts of interest in this work.

\section{References}

1. Weiss H, Wasserheit J, Barnabas R, Hayes R, Abu-Raddad L. Persisting with prevention: the importance of adherence for HIV prevention. Emerg Themes Epidemiol. 2008;5:8.

2. Colfax G, Buchbinder S, Vamshidar G, et al. Motivations for participation in an HIV vaccine efficacy trial. J Acquir Immune Defic Syndr. 2005;39:359-364

3. Tharawan K, Manopaiboon C, Ellertson C, et al. Women's willingness to participate in microbicide trials in Northern Thailand. J Acquir Immune Defic Syndr. 2001;28:180-186.

4. Kenyon S, Dixon-Woods M, Jackson C, Windridge K, Pitcheforth E. Participating in a trial in a critical situation: a qualitative study in pregnancy. Qual Saf Health Care. 2006;15:98-101.

5. Shaffer D, Yebei V, Ballidawa J, et al. Equitable treatment for HIV/ AIDS clinical trial participants: a focus group study of patients, clinical researchers, and administrators in western Kenya. J Med Ethics. 2006;32: $55-60$. 
6. Padian N, Buve A, Balkus J, Serwadda D, Cates W Jr. Biomedical interventions to prevent HIV infection: evidence, challenges and way forward. Lancet. 2008;372:585-599.

7. Watson-Jones D, Baisley K, Rusizoka M, et al. Measurement and predictors of adherence in a trial of HSV suppressive therapy in Tanzania. Contemp Clin Trials. 2009;30:504-512.

8. Mauck CK, Straten A. Using objective markers to assess participant behavior in HIV prevention trials of vaginal microbicides. J Aquir Human Immune Defic Syndr. 2008;49:64-69.

9. Delany S, Mlaba N, Clayton T, et al. Impact of aciclovir on genital and plasma HIV-1 RNA in HSV-2/HIV-1 co-infected women: a randomized placebo-controlled trial in South Africa. AIDS. 2009;23:461-469.

10. Pope S, Ziebland S, Mays N. Qualitative research in health care: analyzing qualitative data. BMJ. 2000;320:114-116.

11. Strauss A, Corbin J. Basics of Qualitative Research: Grounded Theory Procedures and Techniques. Thousand Oaks, CA: Sage Publications; 1990.

12. Straus SE, Takiff HE, Seidlin M, et al. Suppression of frequently recurring genital herpes. A placebo-controlled double-blind trial of oral acyclovir. $N$ Engl J Med. 1984;310:1545-1550.

13. Douglas JM, Critchlow C, Benedetti J, et al. A double-blind study of oral acyclovir for suppression of recurrences of genital herpes simplex virus infection. N Engl J Med. 1984;310:1551-1556.

14. Gupta A, Wald A, Krantz E, et al. Valacyclovir and acyclovir for suppression of shedding of herpes simplex virus in the genital tract. J Infect Dis. 2004;190:1374-1381.

15. Delany S, Mlaba N, Clayton T, et al. Impact of aciclovir on genital and plasma HIV-1 RNA in HSV-2/HIV-1 co-infected women: a randomized placebo-controlled trial in South Africa. AIDS. 2009;23:461-469.
16. Jewkes R, Abrahams N, Mvo Z. Why do nurses abuse patients? Reflections from South African obstetric services. Soc Sci Med. 1998;47:1781-1795.

17. Wood K, Jewkes R. Blood blockages and scolding nurses: barriers to adolescent contraceptive use in South Africa. Reprod Health Matters. 2006;14:109-118.

18. Stadler JJ, Delany S, Mntambo M. Women's perceptions and experiences of HIV prevention trials in Soweto, South Africa. Soc Sci Med. 2008;66:189-200.

19. Stirratt M, Christopher C. Adherence to biomedical HIV prevention methods: considerations drawn from HIV treatment adherence research. Curr HIV/AIDS Rep. 2008;5:186-192.

20. Ross S, Grant A, Counsell C, Gillespie W, Russell I, Prescott R. Barriers to participation in randomised controlled trials: a systematic review. J Clin Epidemiol. 1999;52:1143-1156.

21. Sarkar N. Barriers to condom use. Eur J Contracept Reprod Health Care. 2008;13:114-122.

22. van der Straten A, Cheng H, Moore J, et al; MIRA Team. The use of the diaphragm instead of condoms in a phase III diaphragm trial. AIDS Behav. 2008;13:564-572.

23. Wendler D, Krohmal B, Emanuel E, Grady C. Why patients continue to participate in clinical research. Arch Intern Med. 2008;168: 1294-1299.

24. Coetzee D, Hildebrand K, Boulle A, et al. Outcomes after two years of providing antiretroviral treatment in Khayelitsha, South Africa. AIDS. 2004;18:887-895.
Patient Preference and Adherence

\section{Publish your work in this journal}

Patient Preference and Adherence is an international, peer-reviewed, open access journal focusing on the growing importance of patient preference and adherence throughout the therapeutic continuum. Patient satisfaction, acceptability, quality of life, compliance, persistence and their role in developing new therapeutic modalities and compounds to

\section{Dovepress}

optimize clinical outcomes for existing disease states are major areas of interest. This journal has been accepted for indexing on PubMed Central. The manuscript management system is completely online and includes a very quick and fair peer-review system. Visit http://www.dovepress.com/ testimonials.php to read real quotes from published authors. 